\title{
An interacting multiple models probabilistic data association algorithm for maneuvering target tracking in clutter
}

\author{
Xingxiu $\mathrm{Li}^{1}$, Panlong $\mathrm{Wu}^{2 *}$ and Xinyu Zhang ${ }^{2}$ \\ 1 Department of Science, Nanjing University of Science and Technology, \\ 210094, Nanjing, China \\ 2 Department of Automation, Nanjing University of Science and \\ Technology, 210094, Nanjing, China \\ *plwu@163.com
}

\begin{abstract}
.
To solve the problem of tracking maneuvering target in the presence of clutter, the debiased converted measurement based interacting multiple model (IMMDCM) estimator in combination with the probabilistic data association (PDA) technique is proposed for airborne target tracking. Under the architecture of the proposed algorithm, the IMM deals with the model switching, the debiased converted measurement filter accounts for non-linearity in the dynamic system models, while the PDA handles data association and measurement uncertainties in clutter. The simulation results show that the proposed algorithm can improve the tracking precision for maneuvering target in clutters, and has higher tracking precision than the traditional IMMEKF based PDA algorithm.
\end{abstract}

Keywords: Target tracking, Debiased converted measurement, Probabilistic data association, Interacting multiple model, Clutter

\section{Introduction}

Maneuvering target tracking is a hot topic in the field of target tracking. The key problem of maneuvering target tracking is to establish an accurate target's movement model and a suitable tracking filtering algorithm. Target's maneuvering refers to the sudden change in unpredictable circumstances, such as performing some sort of tactic actions, including acceleration, deceleration, 
steering and so on. Now, the interacting multiple model (IMM) algorithm is widely used for maneuvering target tracking [1].

Combining IMM with appropriate data association algorithm can realize maneuvering target tracking in clutters [2,3], such as ML-PDA, IMMPDA, IMM-MHT and so on. A ML-PDA algorithm has been shown to be robust in a cluttered environment for a constant velocity target. However, it cannot be applied to the situation where targets undergo maneuvers. An adaptive update rate tracking algorithm based on modified IMM-PDA is proposed to avoid tracking loss of maneuvering target tracking in clutters [4]. An IMMPDAF algorithm was proposed to support the navigation and surveillance services of the air traffic management system [5]. In order to gain possible improvement on the tracking performance, the debiased converted measurement based interacting multiple model (IMMDCM) estimator is combined with the PDA filter to create an IMMDCMPDA filter for airborne target tracking.

\section{Radar Data Debiased Converted Measurement}

The measured target position ( the distance $r_{m}$, the azimuth $\beta_{m}$ and the elevation $\theta_{m}$ ) is defined with respect to the true position ( the true distance $r$, the true azimuth $\beta$ and the true elevation $\theta$ ) as:

$$
\left\{\begin{array}{l}
r_{m}=r+\tilde{r} \\
\theta_{m}=\theta+\tilde{\theta} \\
\beta_{m}=\beta+\tilde{\beta}
\end{array}\right.
$$

where the errors in distance $\tilde{r}$, azimuth $\tilde{\beta}$ and elevation $\tilde{\theta}$ are assumed to be independent with zero mean and standard deviation $\sigma_{r}, \sigma_{\beta}$ and $\sigma_{\theta}$ respectively. These polar measurements are converted to the Cartesian coordinate measurements

$$
Z=\left[\begin{array}{c}
x_{m} \\
y_{m} \\
z_{m}
\end{array}\right]=\left[\begin{array}{c}
r_{m} \cos \theta_{m} \cos \beta_{m} \\
r_{m} \cos \theta_{m} \sin \beta_{m} \\
r_{m} \sin \theta_{m}
\end{array}\right]
$$


If the measurement errors of $r, \beta$ and $\theta$ are small and target distance is close, errors statistic approximations obtained in Cartesian coordinates are accurate. These approximations are obtained by taking the first-order terms of a Taylor series expansion for the (2) to approximate the Cartesian coordinate errors as

$$
\begin{aligned}
& \tilde{x}=\tilde{r} \cos \theta \cos \beta-\tilde{\theta} r \sin \theta \cos \beta-\tilde{\beta} r \cos \theta \sin \beta \\
& \tilde{y}=\tilde{r} \cos \theta \sin \beta-\tilde{\theta} r \sin \theta \sin \beta+\tilde{\beta} r \cos \theta \cos \beta \\
& \tilde{z}=\tilde{r} \sin \theta+\tilde{\theta} r \cos \theta
\end{aligned}
$$

the average true deviation $\mu_{a}$ and average true covariance $R_{a}$ of converted measurement can be found in [6]. When measurement in the spherical coordinate is converted to be in Cartesian coordinate, the measurement is modified as

$$
Z_{c}=Z-\mu_{a}=\left[\begin{array}{l}
r_{m} \cos \eta_{m} \cos \theta_{m} \\
r_{m} \cos \eta_{m} \sin \theta_{m} \\
r_{m} \sin \eta_{m}
\end{array}\right]-\mu_{a}
$$

\section{IMMDCMPDA Algorithm Principle}

Supposing there are $r$ model-matched filters used by IMMDCM-PDA. One complete cycle of the proposed IMMDCM-PDA algorithm is given as follows

(1) Mixing probabilities calculation.

$$
\mu_{i j}(k-1)=\frac{1}{C_{j}} p_{i j} \mu_{i}(k-1), C_{j}=\sum_{i=1}^{r} p_{i j} \mu_{i}(k-1), i, j=1,2 \cdots r .
$$

where $\mu_{j}(k-1)$ is the conditional probability of the model $j$ at time $k-1, C_{j}$ is the normalizing constant. $\mu_{i j}(k-1)$ is the mixing probability, which denotes the probability of system switches from model $i$ to model $j$ at $k-1 . p_{i j}$ is the transition probability matrix.

(2) Model filtering in clutter. The DCMPDA is used for each model to calculate the model-conditioned state estimates and covariance.

(a) Input interaction. Computing the input state and covariance matrices for the $r$ filters

$$
\hat{X}_{j}(k-1)=\sum_{i=1}^{r} \mu_{i j}(k-1) X_{i}(k-1) \quad j=1,2 \cdots r
$$




$$
\begin{gathered}
\hat{P}_{j}(k-1)=\sum_{i=1}^{r} \mu_{i j}(k-1) \times\left\{\left[X_{i}(k-1)-\hat{X}_{i}(k-1)\right]\left[X_{i}(k-1)-\hat{X}_{i}(k-1)\right]^{T}+P_{i}(k-1)\right\} \\
j=1, \ldots ., r
\end{gathered}
$$

(b) Converted measurement error calculation. Calculating the $\mu_{a}$ and $R_{a}$.

(c) Model filtering. Computing the DCMPDA filter for $r$ filters.

$$
\hat{X}_{j}(k \mid k-1)=F_{j}(k-1) \hat{X}_{j}(k-1)
$$

$$
\begin{aligned}
& \hat{P}_{j}(k \mid k-1)=F_{j}(k-1) \hat{P}_{j}(k-1) F_{j}^{T}(k-1)+G_{j}(k-1) Q_{j}(k-1) G_{j}^{T}(k-1) \\
& \hat{z}_{j}(k \mid k-1)=H_{j}(k) \hat{X}_{j}(k \mid k-1) \\
& S_{j}(k)=H_{j}(k-1) P_{j}(k \mid k-1) H_{j}^{T}(k-1)+R_{j a}(k-1) \\
& \hat{P}_{j}(k)=\hat{P}_{j}(k \mid k-1)-K_{j}(k) S_{j}(k) K_{j}^{T}(k) \\
& \hat{X}_{j}(k)=\hat{X}_{j}(k \mid k-1)+K_{j}(k) \tilde{v}_{j}(k)
\end{aligned}
$$

Where $m_{k}$ is the number of validated measurements. $\tilde{v}(k)=\sum_{i=1}^{m_{k}} \beta_{i}(k) v_{i}(k)$,

$$
\begin{aligned}
& v_{j}(k)=Z(k)-\mu_{j a}(k)-H_{j}(k) \hat{X}_{j}(k \mid k-1), \\
& \beta_{j}(k)=\frac{e_{j}(k)}{b(k)+\sum_{j=1}^{m_{k}} e_{j}(k)}, j=1,2, \ldots, m_{k}, \beta_{0}(k)=\frac{b(k)}{b(k)+\sum_{j=1}^{m_{k}} e_{j}(k)}, \\
& b(k)=\lambda(2 \pi)^{n_{2} / 2}|S(k)|^{1 / 2}\left(1-P_{D} P_{G}\right) / P_{D}, e_{j}(k)=\exp \left[-\frac{1}{2} v_{j}^{T}(k) S^{-1}(k) v_{j}(k)\right],
\end{aligned}
$$

$H_{j}(k)$ is debiased converted measurements equation.

(3) Model update in clutter.

$$
\mu_{j}(k)=\frac{1}{\sum_{j=1}^{r} \Lambda_{j}(k) C_{j}} \Lambda_{j}(k) C_{j}
$$

$\Lambda_{j}(k)$ is the likelihood of the jth measurement for jth PDA filter, 
$\Lambda_{j}(k)=\frac{P_{D} V_{j}^{-m_{k}+1}(k)}{m_{k}}\left[b_{j}(k)+\sum_{i=1}^{m_{k}} e_{j, i}(k)\right] . \mathrm{P}_{\mathrm{D}}$ and $\mathrm{P}_{\mathrm{G}}$ are the target detection probability and the gate probability, respectively. $V_{j}(k)$ is the volume of the ellipse tracking gate.

$V_{j}(k)=c_{n_{z}} \gamma^{n_{2} / 2}\left|S_{j}(k)\right|^{1 / 2}$

$n_{z}$ is number of total validated measurements, the coefficient $c_{n_{z}}$ depends on the dimension of $n_{z}$.

$\beta_{i}(k)$ is the association probability of the validated measurements. $\beta_{0}(k)$ is the probability that all measurements are not valid.

(4) State Combination.

$$
X(k)=\sum_{j=1}^{r} \hat{X}_{j}(k) \mu_{j}(k)
$$$$
P(k)=\sum_{j=1}^{r}\left\{P_{j}(k \mid k)+\left[X(k)-\hat{X}_{j}(k)\right]\left[X(k)-\hat{X}_{j}(k)\right]^{T}\right\} \mu_{j}(k)
$$

\section{Simulation and Results}

In this paper, the CV model and Singer model are selected to build in the IMMDCMPDA algorithm. The target dynamic model can be found in [7]. The following example of tracking a highly maneuvering airborne target is considered. The sampling rate is $T=0.1 \mathrm{~s}$. The relative trajectory of target and aircraft platform is shown in Fig.1. Fig.2, Fig.3 and Fig.4 show the position estimation error of two algorithms, respectively. 

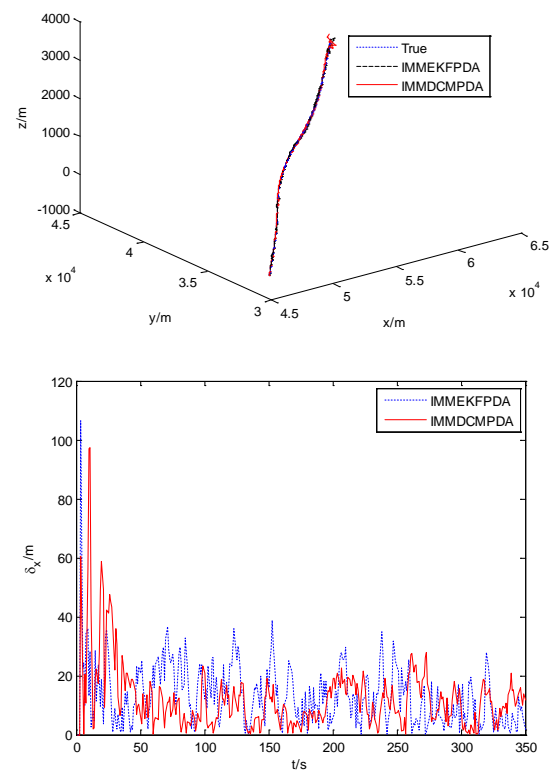

Fig. 1 The comparison of trajectory Fig.2 The comparison of $x$ error
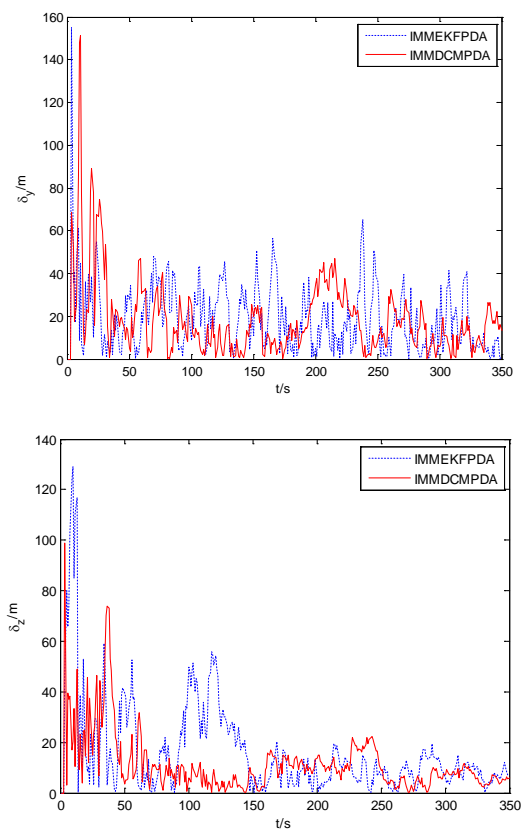

Fig.3 The comparison of y error

Fig.4 The comparison of $\mathrm{z}$ error 


\section{Conclusions}

In this paper, a novel IMMDCMPDA algorithm is proposed, which is capable of adaptively tracking the maneuvering airborne target in clutter. By abandoning the Kalman filter framework and using DCM filter in the proposed algorithm, the good tracking precision is achieved without increasing the computational complexity. The IMMDCMPDA algorithm achieves a better tracking performance compared with the IMMEKFDA. Simulation results show the effectiveness of the proposed algorithm for maneuvering target tracking in clutters.

\section{Acknowledgement}

This work was supported by the National Natural Science Foundation of China (61104196, 61301217, 61473153), Research Fund for the Doctoral Program Higher Education of China (20123219120043), Natural Science Foundation of Jiangsu Province (BK20131352) and “Zijin star” Funding.

\section{References}

[1] P. L. Wu, L. Z. and X. Y. Zhang: The design of DSP/FPGA based maneuvering target tracking system. WSEAS Transactions on Circuits and Systems,Vol. 13 (2014), p.75

[2] D. Musicki and S. Suvorova: Tracking in clutter using IMM-IPDA-based algorithms. IEEE Transactions on Aerospace and Electronic Systems, Vol. 44 (2008), p.111

[3] S. S. Blackman: Multiple hypothesis tracking for multiple target tracking. IEEE Trans. on Aerospace and Electronic System, Vol.19 (2004), p.5

[4] T. Chen, Z. S. He and Y. X. Li: Adaptive update rate tracking based on modified IMM-PDA. IEEE CIE International Conference on Radar, (2011) 
[5] S. S. Jan and Y. C. Kao: Radar tracking with an interacting multiple model and probabilistic data association filter for civil aviation applications. Sensors, Vol.13 (2013), p. 6636

[6] L. Z. Zhang, P. L. Wu and X. Y. Zhang: Air defense missile detonation delay control Based on FPGA/DSP[J]. WSEAS Transactions on System, Vol.12 (2013), p. 202

[7] P. L. Wu, B. B. Wang and C. H. Ji: Design and realization of short range defence radar target tracking system based on DSP/FPGA. WSEAS Transactions on System, Vol.10 (2011), p.376 